\title{
53. DATA REPORT: NANNOFOSSIL BIOHORIZONS AND AGE-DEPTH PLOTS FOR LEG 133 SITES ${ }^{1}$
}

\author{
S. Gartner ${ }^{2}$ and W. Wei ${ }^{3}$
}

Paleontological studies conducted subsequent to the completion of Leg 133 led to refinements of the biostratigraphy for the Leg 133 sites. These biostratigraphic refinements bear on the calculations of sedimentation rates and on the age-depth plots prepared for the Initial Reports volume for Leg 133. To make available the revised data to anyone who may wish to make use of it, the revised biostratigraphic information is presented here in tabulated form (Tables 1 and 2). Revised age-depth plots also are presented for all of the sites (Figs. $1-13$ ) to facilitate comparison of sedimentation rate curves and to identify intervals where significant changes have been made based on post-cruise studies. The revised age-depth plots include calcareous nannofossils only, and the revised data have been taken from the chapters contributed for this volume (Gartner et al., this volume; Wei and Gartner, this volume). Planktonic foraminifer biostratigraphy revisions became available subsequently and could not be readily incorporated. The age-depth plots for Sites 812 through 818 were made with the (ADP) program provided to ODP by Dave Lazarus.

Figures 1 through 6 represent Sites 811 and 819 through 823, which are from the northern transect (Queensland Trough transect). Figures 7 through 13 represent Sites 812 through 818 , which are from the southern transect (Townsville Trough transect).
The labels on the points of the age-depth plots are the plot codes of biohorizons. The names for the biohorizons are given in Tables 1 and 2. The ages for the biohorizons were mainly taken from Berggren et al. (1985), with some modifications where better-constrained ages were available from more recent studies (see Gartner, Wei, and Shuy, this volume; and Wei and Gartner, this volume).

\section{REFERENCE}

Berggren, W.A., Kent, D.V., and Van Couvering, J.A., 1985. The Neogene: Part 2. Neogene geochronology and chronostratigraphy. In Snelling, N.J. (Ed.), The Chronology of the Geological Record. Geol. Soc. London Mem., 10:211-260.

- Abbreviations for names of organizations and publication titles in ODP reference lists follow the style given in Chemical Abstracts Service Source Index (published by American Chemical Society).

Date of initial receipt: 3 August 1992

Date of acceptance: 19 January 1993

Ms 133SR-275

\footnotetext{
'McKenzie, J.A., Davies, P.J., Palmer-Julson, A., et al., 1993. Proc. ODP, Sci. Results, 133: College Station, TX (Ocean Drilling Program).

${ }^{2}$ Department of Oceanography, Texas A\&M University, College Station, TX 77843. U.S.A.

${ }^{3}$ Scripps Institution of Oceanography, University of California at San Diego, La Jolla, CA 92093, U.S.A.
} 


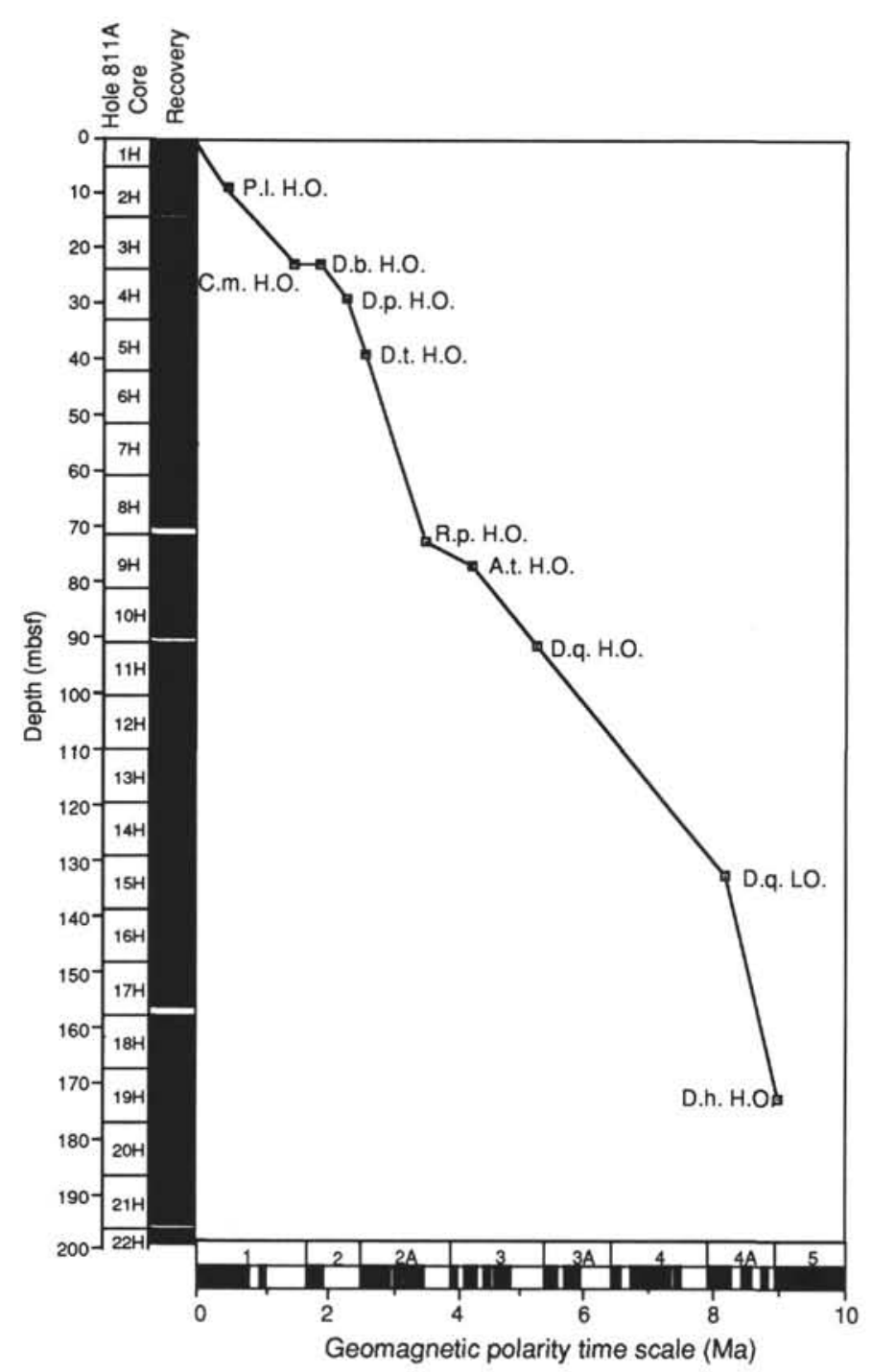

Figure 1. Age vs. depth plot for Hole 811A.

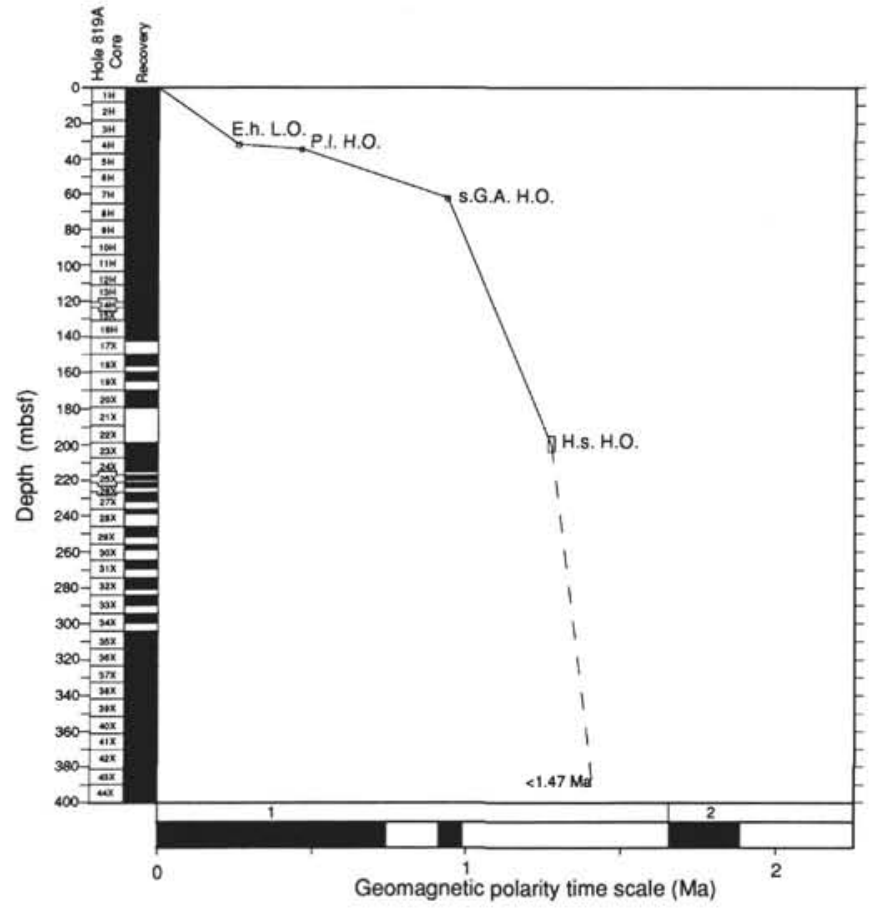

Figure 2. Age vs. depth plot for Hole 819A.

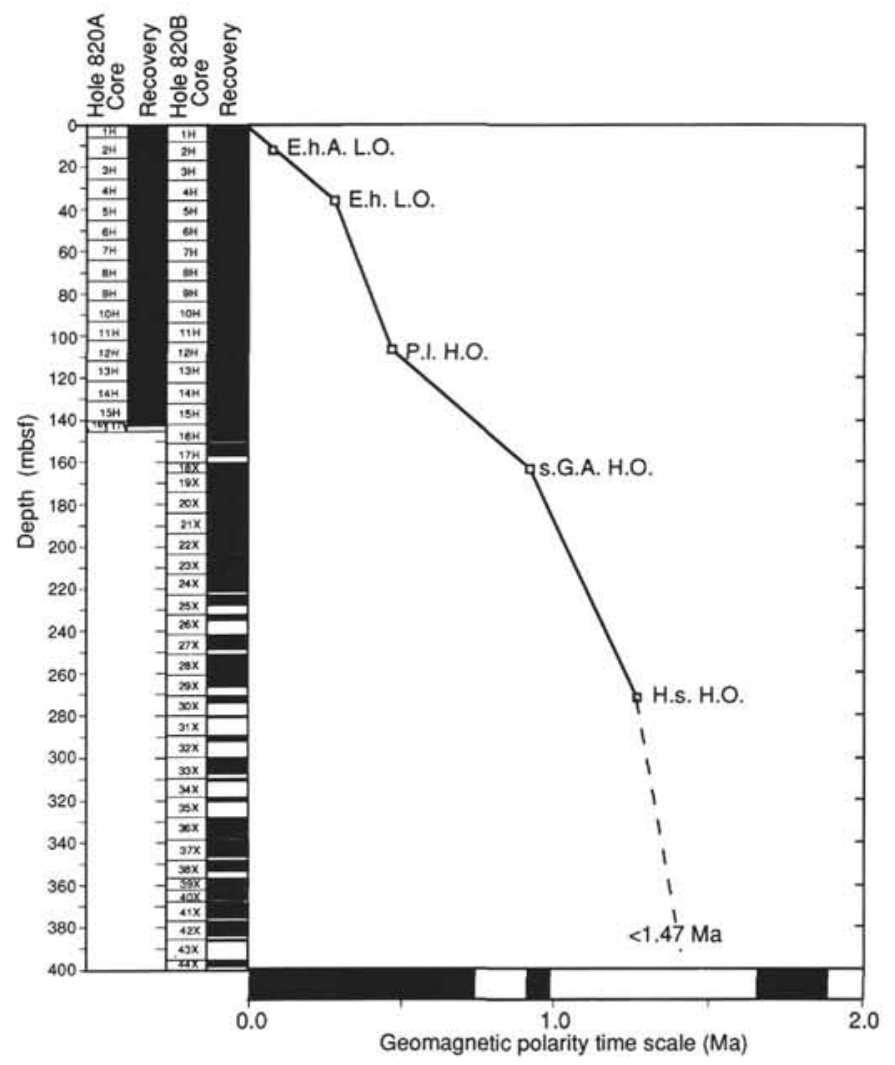

Figure 3. Age vs. depth plot for Holes $820 \mathrm{~A}$ and $820 \mathrm{~B}$. 

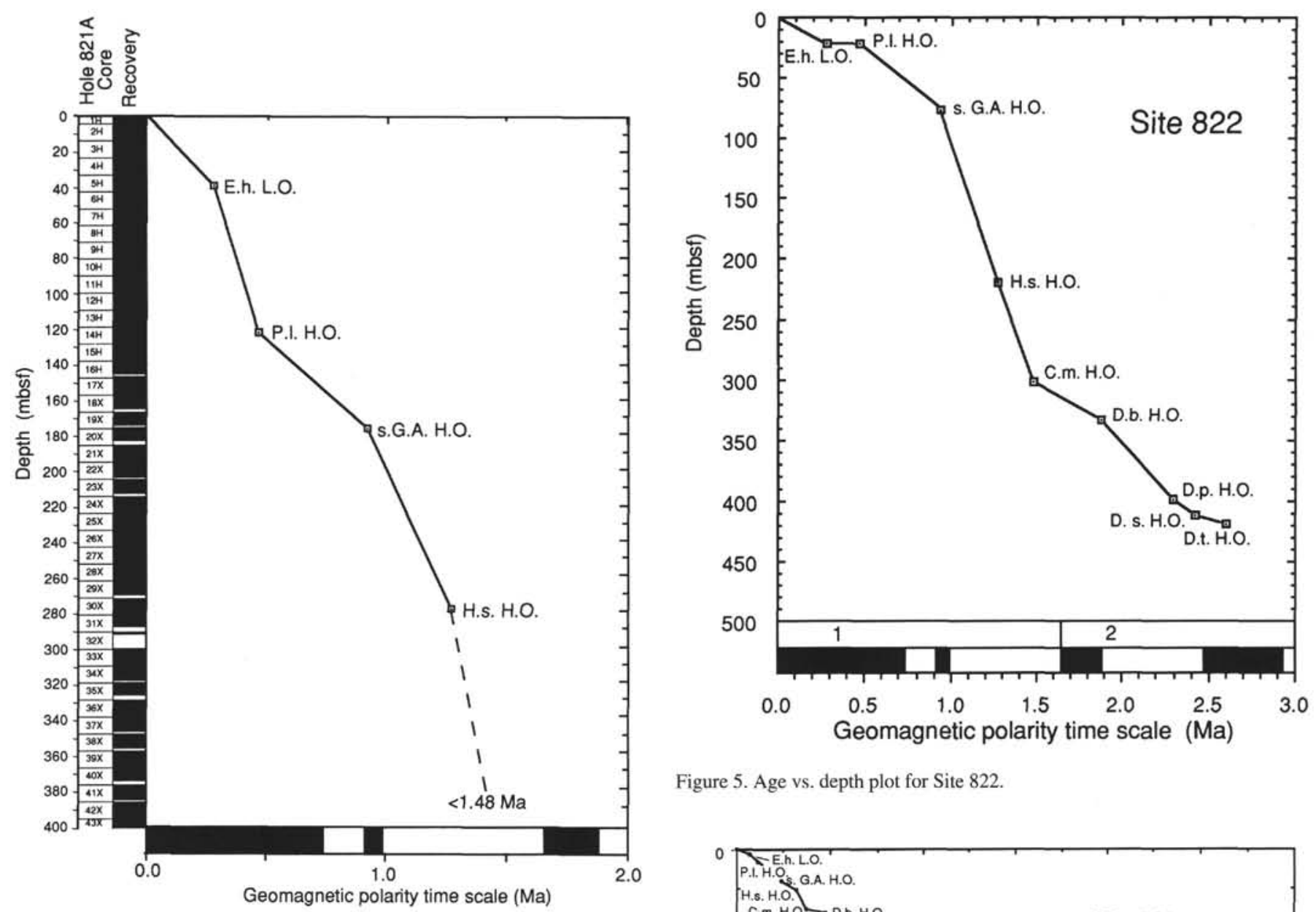

Figure 5. Age vs. depth plot for Site 822 .

Figure 4. Age vs. depth plot for Hole 821A.

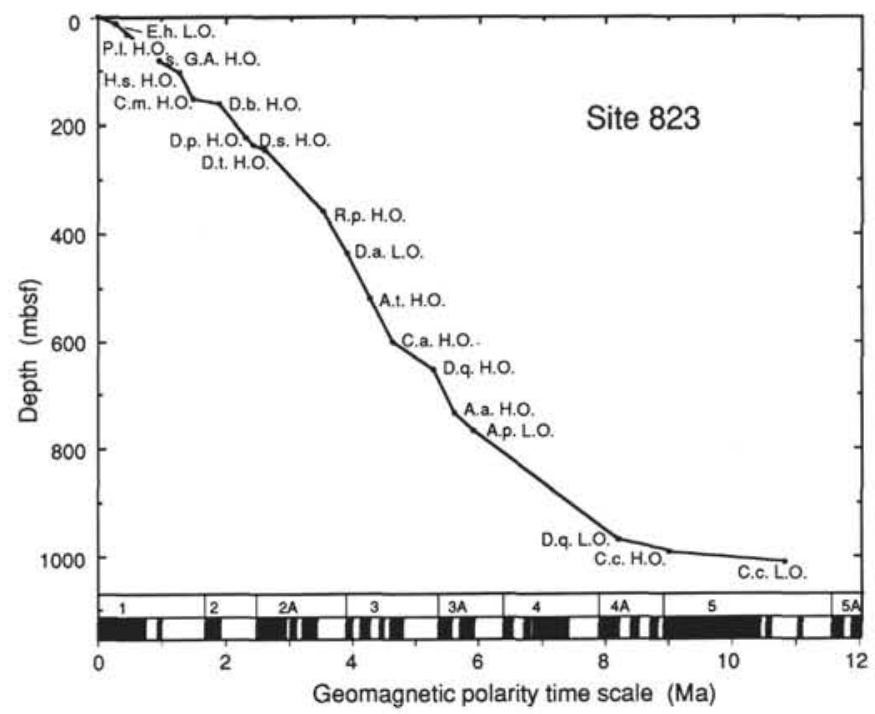

Figure 6. Age vs. depth plot for Site 823. 


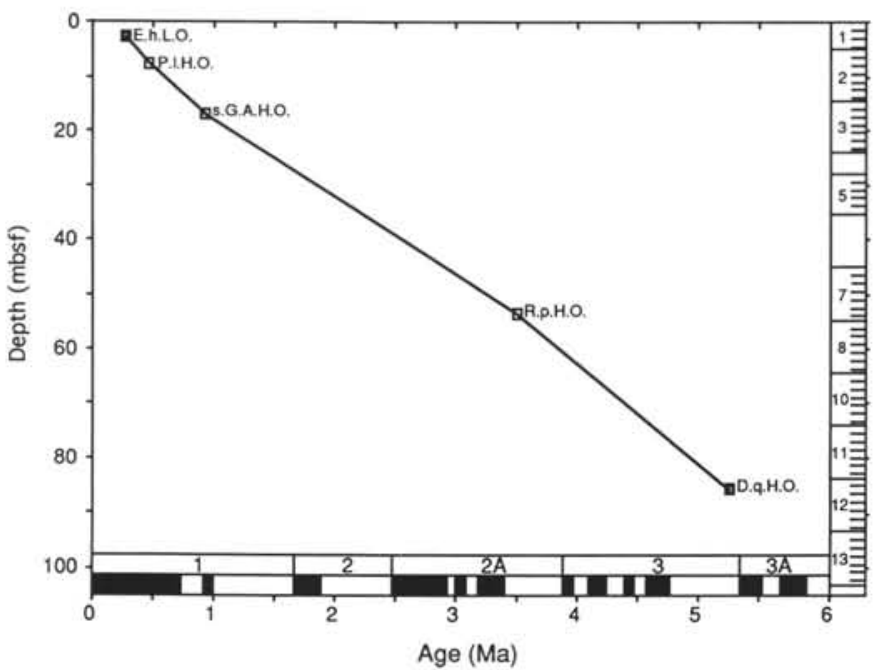

Figure 7. Age vs. depth plot for Site 812.

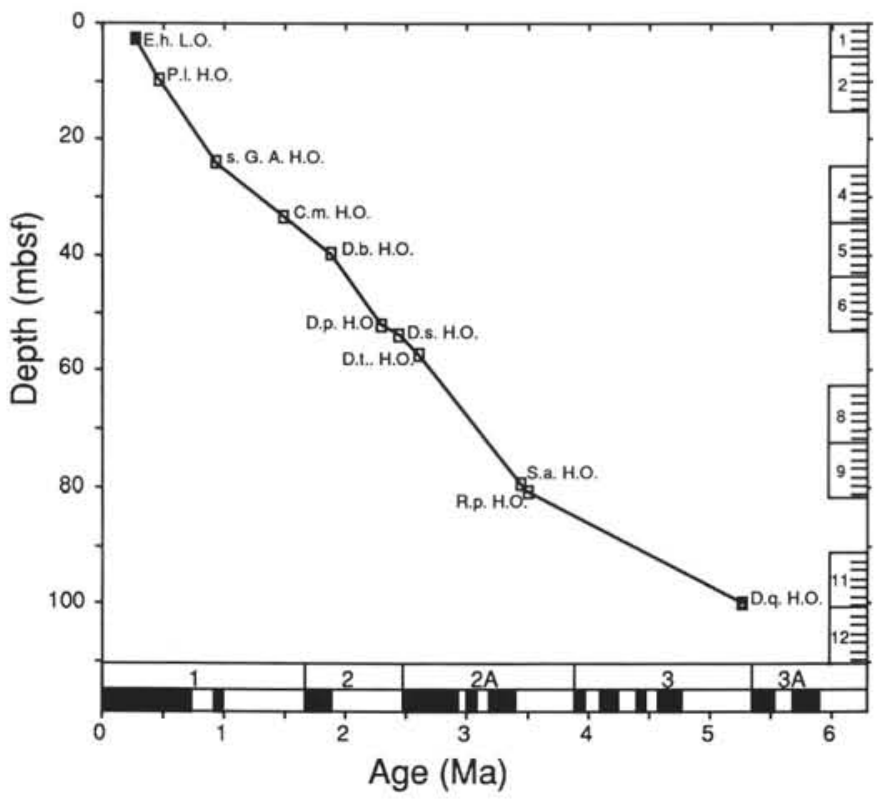

Figure 8. Age vs. depth plot for Hole 813A.

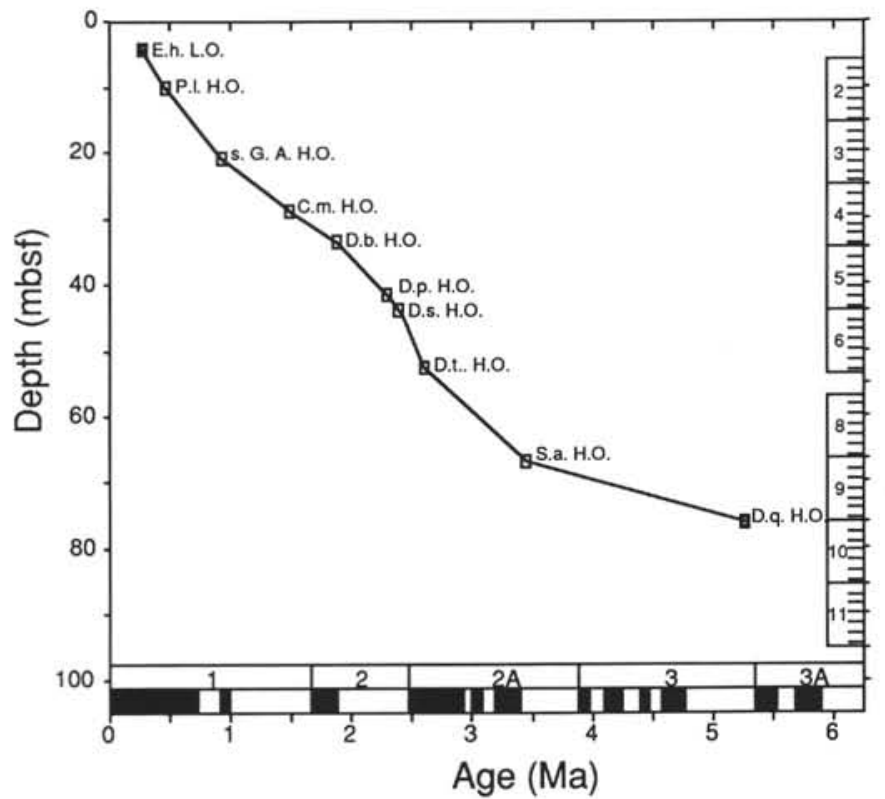

Figure 9. Age vs. depth plot for Hole 814A.

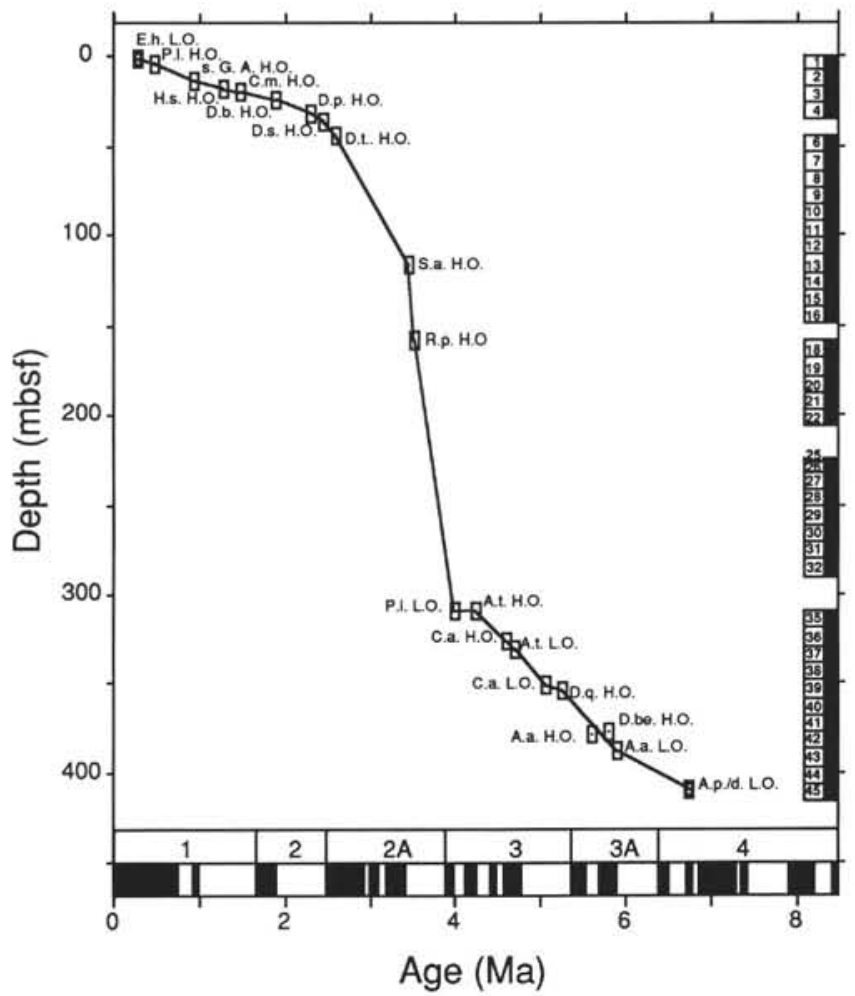

Figure 10. Age vs. depth plot for Hole 815A. 

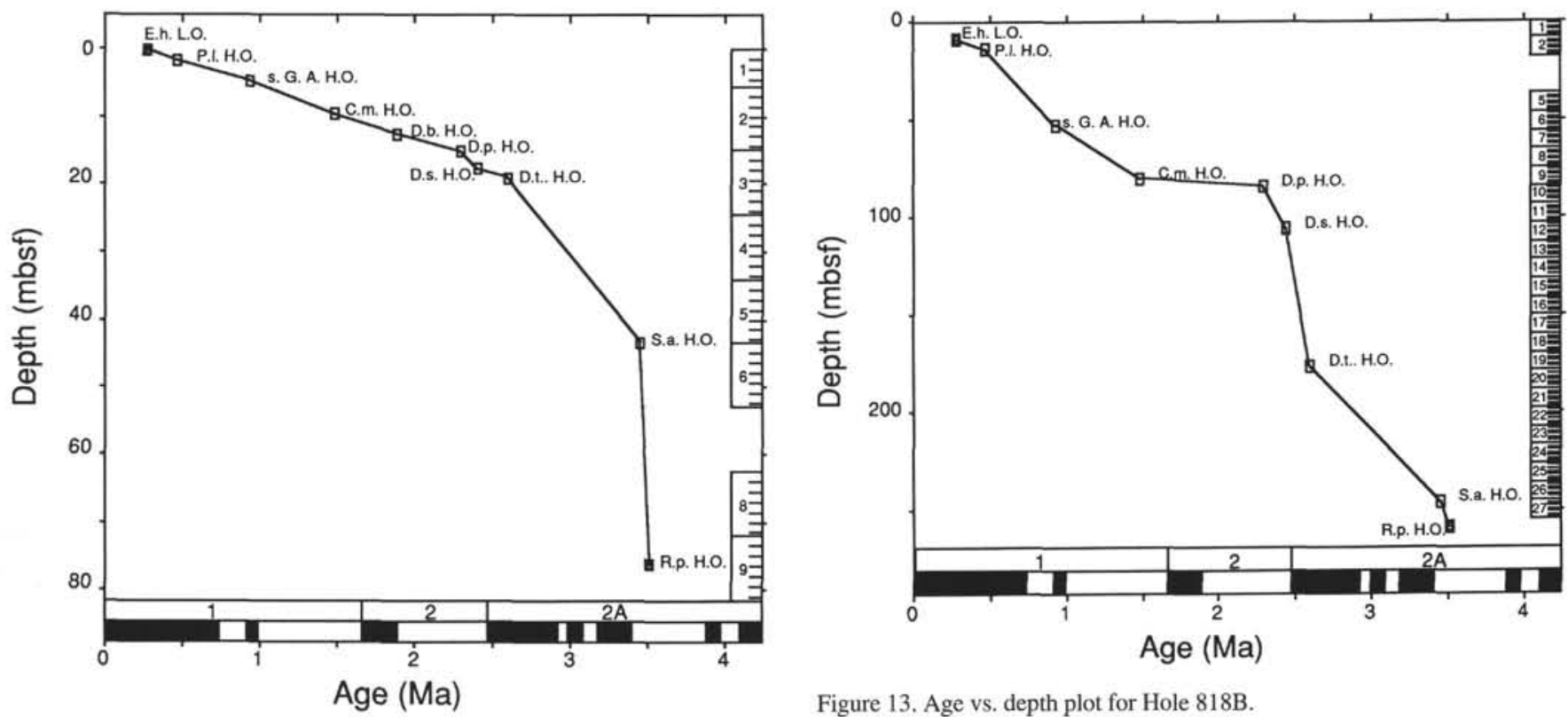

Figure 11. Age vs. depth plot for Hole 816A.

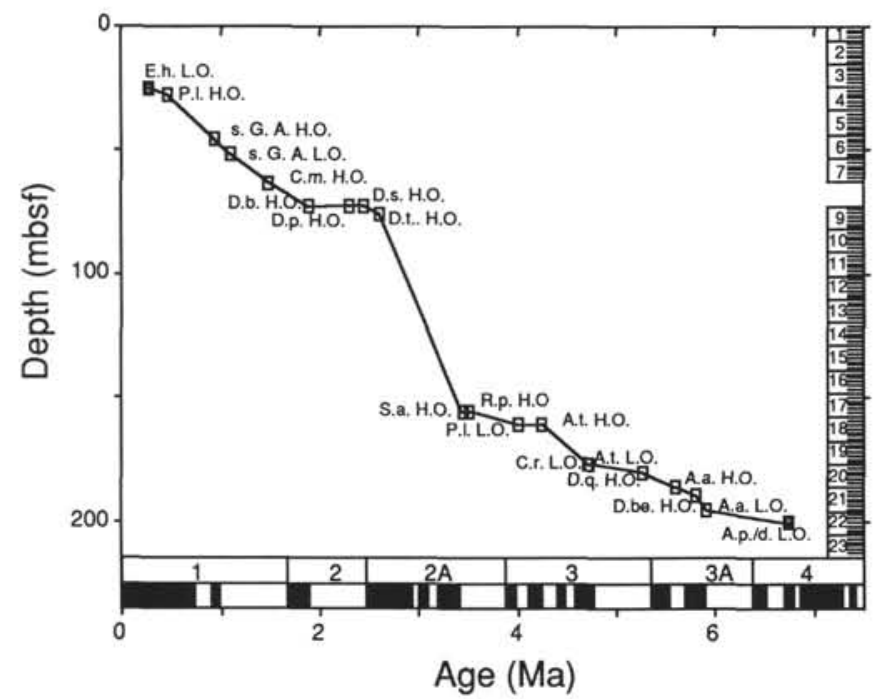

Figure 13. Age vs. depth plot for Hole 818B.

Figure 12. Age vs. depth plot for Hole 817A. 
Table 1. Nannofossil biohorizons for Sites 811 and 819 through 825 .

\begin{tabular}{|c|c|c|c|c|c|c|c|c|c|c|}
\hline \multirow[b]{2}{*}{ Biohorizon } & \multirow[b]{2}{*}{ Plot code } & \multirow[b]{2}{*}{ Age (Ma) } & \multicolumn{8}{|c|}{ Site } \\
\hline & & & 811 & 819 & 820 & 821 & 822 & 823 & 824 & 825 \\
\hline E. huxleyi acme LO & E.h.A L.O. & 0.075 & & 8.4 & 12.1 & & & & & \\
\hline E. huxleyi LO & E.h. L.O. & 0.275 & & 29.4 & 35.8 & 39.3 & 21.8 & 12.2 & & \\
\hline P. lacunosa $\mathrm{HO}$ & P.I. H.O. & 0.465 & 8.9 & 32.4 & 107.1 & 123.1 & 21.8 & 32.0 & 47.8 & \\
\hline small Gephyr, top & s.G.A. H.O & 0.92 & & 56.1 & 163.6 & 176.8 & 77.8 & 80.2 & & \\
\hline H. sellii НО & H.s. H.O. & 1.27 & & 189.2 & 271.7 & 279.2 & 220.0 & 105.7 & & \\
\hline C. macintyrei $\mathrm{HO}$ & C.m. H.O. & 1.48 & 22.9 & $>400$ & $>400$ & $>400$ & 301.5 & 150.9 & 92.8 & \\
\hline D. brouweri $\mathrm{HO}$ & D.b. H.O. & 1.88 & 22.9 & & & & 333.4 & 163.0 & & \\
\hline D. pentaradiatus $\mathrm{HO}$ & D.p. H.O. & 2.29 & 29.4 & & & & 399.3 & 222.3 & & \\
\hline D. surculus $\mathrm{HO}$ & D.s. H.O. & 2.42 & & & & & 411.0 & 236.2 & & \\
\hline D. tamalis $\mathrm{HO}$ & D.t. H.O. & 2.60 & 38.9 & & & & & 244.3 & & \\
\hline R. pseudoumbilicus $\mathrm{HO}$ & R.p. H.O. & 3.51 & 72.6 & & & & & 359.3 & & \\
\hline D. asymmetricus Ac.LO & D.a.A. L.O. & 3.88 & & & & & & 435.5 & & \\
\hline A, tricorniculatus $\mathrm{HO}$ & A.t. H.O. & 4.24 & 76.9 & & & & & 521.6 & & \\
\hline C. armatus $\mathrm{HO}$ & C.a.H.O. & 4.60 & & & & & & 602.0 & & \\
\hline D. quinqueramus $\mathrm{HO}$ & D.q. H.O. & 5.26 & 91.6 & & & & & 653.0 & & \\
\hline A. amplificus $\mathrm{HO}$ & A.a. H.O. & 5.6 & & & & & & 733.1 & & \\
\hline A. amplificus LO & A.a. L.O. & 5.9 & & & & & & 767.6 & & \\
\hline D. quinqueramus LO & D.q. L.O. & 8.2 & 132.6 & & & & & 967.4 & & \\
\hline D. hamatus HO & D.h. H.O. & 8.85 & 172.6 & & & & & & & \\
\hline C. coalitus $\mathrm{HO}$ & C.c. H.O. & 9.0 & & & & & & 989.8 & & \\
\hline C. coalitus LO & C.c. L.O. & 10.8 & & & & & & 1009.1 & & \\
\hline C. floridanus $\mathrm{HO}$ & C.f. H.O. & 11.0 & & & & & & & & 214.3 \\
\hline
\end{tabular}

Table 2. Nannofossil biohorizons for Sites 812 through 818.

\begin{tabular}{|c|c|c|c|c|c|c|c|c|c|c|}
\hline Species & Plot code & $\begin{array}{l}\text { Age } \\
\text { (Ma) }\end{array}$ & $\begin{array}{c}\text { Depth } / 812 \mathrm{~A} \\
\text { (mbsf) }\end{array}$ & $\begin{array}{r}812 \mathrm{C} \\
\text { (mbsf) }\end{array}$ & $\begin{array}{r}813 \mathrm{~A} \\
(\mathrm{mbsf})\end{array}$ & $\begin{array}{r}814 \mathrm{~A} \\
\text { (mbsf) }\end{array}$ & $\begin{array}{r}815 \mathrm{~A} \\
\text { (mbsf) }\end{array}$ & $\begin{array}{r}816 \mathrm{~A} \\
(\mathrm{mbsf})\end{array}$ & $\begin{array}{r}817 \mathrm{~A} \\
\text { (mbsf) }\end{array}$ & $\begin{array}{r}818 \mathrm{~B} \\
\text { (mbsf) }\end{array}$ \\
\hline E. huxleyi LO & E.h. LO. & 0.275 & 2.67 & & 2.63 & 4.12 & 1.1 & 0.37 & 25.07 & 9.53 \\
\hline P. lacunosa $\mathrm{HO}$ & P.I. H.O. & 0.465 & 7.52 & & 9.83 & 10.01 & 4.1 & 1.87 & 28.07 & 14.03 \\
\hline small Gephyrocapsa Acme top & s.G.A.H.O. & 0.93 & 17.02 & & 23.82 & 21.01 & 13.9 & 4.87 & 45.56 & 53.51 \\
\hline small Gephyrocapsa Acme bottom & s.G.A. H.O. & 1.1 & & & & & 17.4 & & 51.56 & \\
\hline H. sellii HO & H.s. H.O. & 1.27 & & & & & 18.9 & & & \\
\hline C. macintyrei $\mathrm{HO}$ & C.t. H.O. & 1.48 & 23.9 & 23.27-24.77 & 33.32 & 29.01 & 20.4 & 9.62 & 63.06 & 80.51 \\
\hline G. oceanica LO & G.o. LO. & 1.59 & & & & & & & & \\
\hline D. brouweri $\mathrm{HO}$ & D.b. H.O. & 1.88 & & 25.9 & 39.81 & 33.51 & 24.9 & 12.62 & 72.56 & \\
\hline D. pentaradiatus $\mathrm{HO}$ & D.p. H.O. & 2.29 & $25.96-27.90$ & $26.85-29.0$ & 52.31 & 41.51 & 31.4 & 15.18 & 72.56 & 84.58 \\
\hline D. surculus $\mathrm{HO}$ & D.s. H.O. & 2.24 & & & 53.81 & 44.51 & 36.4 & 17.62 & 72.56 & 106.01 \\
\hline D. asymmetricus $\mathrm{HO}$ & D.a. H.O. & 2.24 & & & & & & & & \\
\hline D. tamalis $\mathrm{HO}$ & D.t. H.O. & 2.6 & & & 57.31 & 52.51 & 44.72 & 19.12 & 75.56 & 177.02 \\
\hline D. variabilis $\mathrm{HO}$ & D.v. H.O. & 2.9 & & & & & & & & \\
\hline S. abies HO & S.a. H.O. & 3.45 & $29.26-35.40$ & & 79.31 & 66.83 & 116.91 & 43.5 & 155.7 & 246.08 \\
\hline R.pseudoumbilicus $\mathrm{HO}$ & R.p. H.O. & 3.51 & & 53.61 & 80.81 & & 158.9 & 76.11 & 155.7 & 259.51 \\
\hline$P$. lacunosa LO & P.I. L.O. & 4 & & & & & 309.46 & & 161.06 & \\
\hline A. tricorniculatus $\mathrm{HO}$ & A.t. H.O. & 4.24 & & & & & 309.46 & & 161.06 & \\
\hline C. armatus $\mathrm{HO}$ & C.a. H.O. & 4.6 & & & & & 326.35 & & & \\
\hline A. tricorniculatus LO & A.t. L.O. & 4.7 & & & & & 331.61 & & 176.7 & \\
\hline C. rugosus LO & C.r. L.O. & 4.72 & & & & & & & 176.7 & \\
\hline C. armatus $\mathrm{LO}$ & C.a. L.O. & 5.06 & & & & & 351 & & & \\
\hline D. quinqueramus $\mathrm{HO}$ & D.q. H.O. & 5.26 & & 85.61 & 99.81 & 76 & 354 & & 180.07 & \\
\hline A. amplificus $\mathrm{HO}$ & A.a. H.O. & 5.6 & & & & & 378.54 & & 186.07 & \\
\hline D. berggreni $\mathrm{HO}$ & D.be. H.O. & 5.8 & & & & & 377.04 & & 189.57 & \\
\hline A. amplificus LO & A.a. LO. & 5.9 & & & & & 387.3 & & 195.57 & \\
\hline A. primus/A. delicatus LO & A.p/d. LO. & 6.74 & & & & & 409.36 & & 200.56 & \\
\hline
\end{tabular}

